\title{
Article
}

\section{Influence of Fermions on Vortices in SU(2)-QCD}

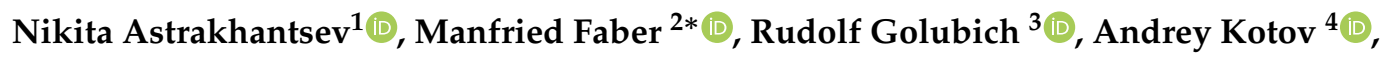 \\ Aleksandr Nikolaev ${ }^{5}$ (i)
}

1 Physik-Institut, Universität Zürich, Winterthurerstrasse 190, CH-8057 Zürich, Switzerland and Institute for Theoretical and Experimental Physics NRC “Kurchatov Institute”, Moscow, 117218 Russia; nikita.astrakhantsev@physik.uzh.ch

2 Atominstitut, Techn. Univ. Wien, Austria; faber@kph.tuwien.ac.at

3 Atominstitut, Techn. Univ. Wien, Austria; rudolf.golubich@gmail.com

4 Bogoliubov Laboratory of Theoretical Physics, Joint Institute for Nuclear Research, Dubna, 141980 Russia; akotov@theor.jinr.ru

5 Department of Physics, College of Science, Swansea University, Swansea SA2 8PP, United Kingdom; aleksandr.nikolaev@swansea.ac.uk

* Correspondence: faber@kph.tuwien.ac.at

\begin{abstract}
Gauge fields control the dynamics of $f$ ermions. On the other hand a back reaction of fermions on the gauge field is expected. This back reaction is investigated within the vortex picture of the QCD vacuum.
\end{abstract}

Keywords: quantum chromodynamics; confinement; center vortex model; influence of fermions

PACS: 11.15.Ha, 12.38.Gc

\section{Introduction}

The QCD vacuum is highly nontrivial and has magnetic properties, as we know since Savvidy's article [1]. The QCD vacuum should explain the non-perturbative properties of QCD, among them confinement and chiral symmetry breaking. Lattice QCD puts the means at our disposal to answer the question about the important degrees of freedom of this non-perturbative vacuum. In the center vortex picture [2] the QCD vacuum is a condensate of closed quantized magnetic flux lines. These flux lines have a random shape and evolve in time and form therefore closed surfaces in dual space. They may expand and shrink, fuse and split and percolate in the confinement phase in all space-time directions. The finite temperature phase transition is characterized by a loss of center symmetry and correspondingly by the loss of percolation in time direction, vortices get static.

Colour electric charges are a source of electric flux according to Gauss's law. The electric flux between opposite colour charges does not like to penetrate this magnetic "medium" and shrinks to the well-known electric fluxtube. On the other hand the magnetic flux does not like to enter the electric flux tube. Therefore, the condensate of magnetic flux is suppressed in the region of the electric string. Since fermions carry color charges their dynamics is controlled by the gauge field. On the other hand the presence of a fermion condensate is expected to suppress the quantized magnetic flux lines, reducing in this way the gluon condensate and therefore the string tension. If, as usual, the lattice spacing is determined via the string tension, it is therefore expected that dynamical fermions lead to an decrease of the lattice spacing. In this article we show first results for the back reaction of dynamical fermions on the structure of vortices within $\mathrm{SU}(2) \mathrm{QCD}$. 


\section{Method and Results}

We study the configurations described in Ref. [3] for chemical potential $\mu=0$ with a tree level improved Symanzik gauge action [4,5]. For the fermionic degrees of freedom we used staggered fermions with an action of the form

$$
S_{F}=\sum_{x, y} \bar{\psi}_{x} M(\mu, m)_{x, y} \psi_{y}+\frac{\lambda}{2} \sum_{x}\left(\psi_{x}^{T} \tau_{2} \psi_{x}+\bar{\psi}_{x} \tau_{2} \bar{\psi}_{x}^{T}\right)
$$

with

$$
M(\mu, m)_{x y}=m a \delta_{x y}+\frac{1}{2} \sum_{v=1}^{4} \eta_{v}(x)\left[U_{x, \nu} \delta_{x+h_{v}, y} e^{\mu a \delta_{v, 4}}-U_{x-h_{v}, v}^{+} \delta_{x-h_{v}, y} e^{-\mu a \delta_{v, 4}}\right],
$$

where $\bar{\psi}, \psi$ are staggered fermion fields, $a$ is the lattice spacing, $m$ is the bare quark mass, and $\eta_{v}(x)$ are the standard staggered phase factors: $\eta_{1}(x)=1, \eta_{v}(x)=(-1)^{x_{1}+\ldots+x_{v-1}}, v=2,3,4$.

For the field configuration without and with fermions we performed direct maximal center gauge [6]. This is the gauge which brings on average each link variable as close as possible to a $Z_{2}$ center element, while preserving the residual $Z_{2}$ gauge invariance

$$
R^{2}=\sum_{x} \sum_{\mu}\left|\operatorname{Tr}\left[U_{\mu}(x)\right]\right|^{2} \quad \text { is maximal. }
$$

Center projection maps then each $\mathrm{SU}(2)$ link variable to the closest $Z_{2}$ center element,

$$
U_{\mu}(x) \rightarrow Z_{\mu}(x) \equiv \text { sign } \operatorname{Tr}\left[U_{\mu}(x)\right] .
$$

The excitations on the projected $Z_{2}$ lattice form on the dual of a four-dimensional Euclidean lattice closed surface-like objects, known as "P vortices." These are thin objects, only one lattice spacing across.
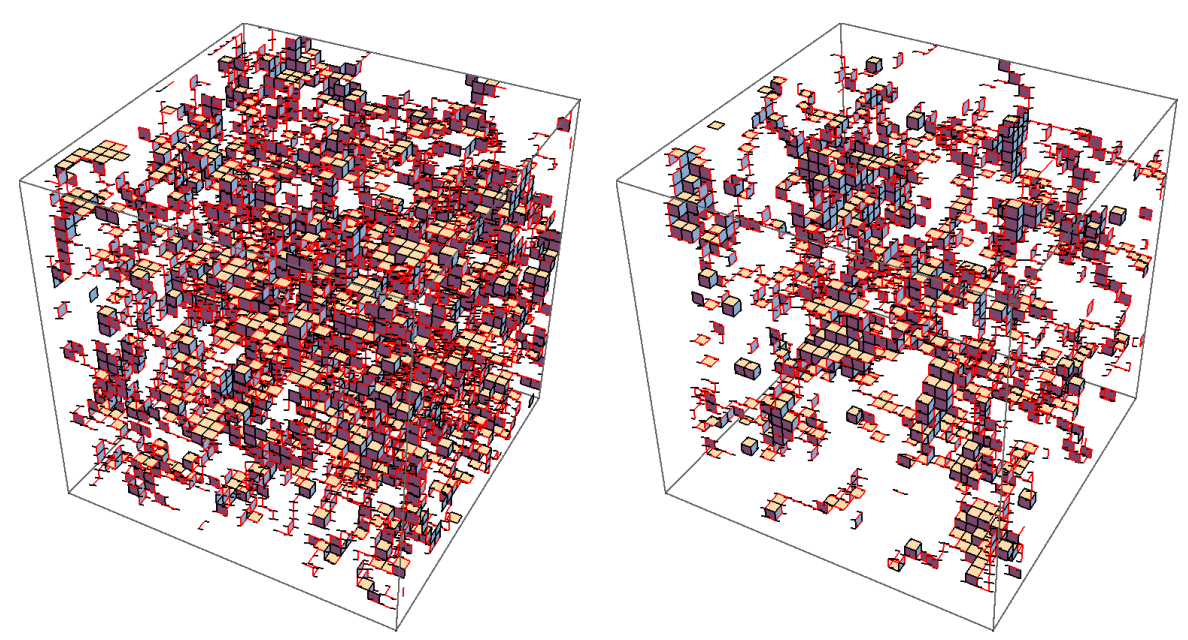

Figure 1. Single time slices of $\mathrm{P}$ vortices on a $32^{4}$ lattice. Both diagrams indicate the $\mathrm{P}$ plaquettes after maximal center gauge and center projection of field configurations at $\beta=1.8$. The left diagram is taken from a pure gluonic configuration and the right diagram from one of the above discussed fermionic configurations.

Examples of P vortices are shown in Fig. 1 of a $32^{4}$ lattice. Since it is difficult to represent surfaces in four dimensions, only time slices of the P vortices are shown. Every P plaquette has four neighbors. If such neighbours are not shown in the diagrams they belong to neighboring time slices. One can clearly see that the $P$ vortices percolate in both diagrams through the whole lattice. The left diagram of Fig. 1 is taken from a pure gluonic configuration at $\beta=1.8$ and the right diagram from a field configuration at the same $\beta$ under the influence of the staggered fermions, as described above. Comparing the two 
diagrams one can clearly see a remarkable suppression of the vortex density by the fermion condensate. The P vortex configurations give an excellent possibility for a lattice determination of the string tension.

Asymptotic values of the Creutz ratios

$$
\chi\left(\frac{r}{a}, \frac{t}{a}\right)=-\ln \frac{\left\langle W\left(\frac{r}{a}+1, \frac{t}{a}+1\right)\right\rangle\left\langle W\left(\frac{r}{a}, \frac{t}{a}\right)\right\rangle}{\left\langle W\left(\frac{r}{a}, \frac{t}{a}+1\right)\right\rangle\left\langle W\left(\frac{r}{a}+1, \frac{t}{a}\right)\right\rangle}
$$

of Wilson loops $W\left(\frac{r}{a}, \frac{t}{a}\right)$ are known to give good values for the string tension, where $r$ and $t$ are the size of the Wilson loop and $a$ is the lattice spacing. We analyze 1000 gluonic and 1000 fermionic configurations of Ref. [3] and show the Creutz ratios for symmetric Wilson loops, with $r=t$, of these two times 1000 configurations in Fig. 2 in dependence of the size of evaluated Wilson loops. In both cases one can clearly see the decrease of the Creutz ratios due to the influence of the Coulomb potential on small Wilson loops. Already at $r=5 a$ the error bars of Creutz ratios $\chi$ from a jackknife analysis are getting sizable and do not really allow to predict the value of asymptotic Creutz ratios and therefore the string tension from the full unprojected configurations.

Creutz ratios from gluonic configurations, $\beta=1.8$

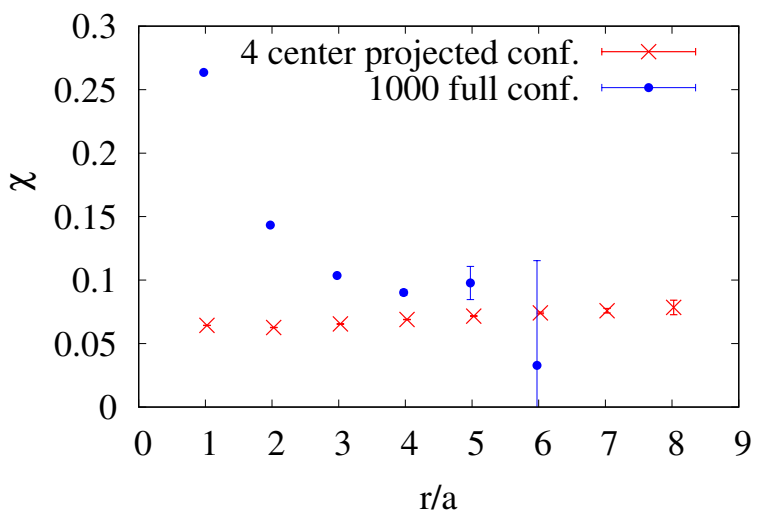

Creutz ratios from fermionic configurations, $\beta=1.8$

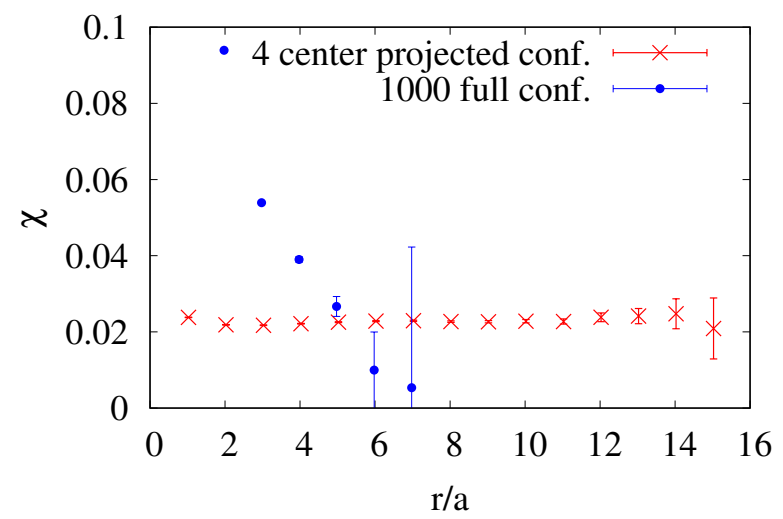

Figure 2. Creutz ratios from full and center projected configurations. Already a few projected configurations give much more precise values for the string tension than 1000 full configurations.

The situation is completely different for Creutz ratios from projected configurations. Already a few configurations give Creutz ratios up to much larger values of $r$. In Fig. 2 the projected Creutz ratios from only four configurations are depicted. They are insensitive to the Coulomb potential and are therefore almost independent of $r$. Some more details about the determination of the projected Creutz ratios are here in order. In Eq. (3) we indicated the gauge functional which we have maximized. This maximization suffers from a Gribov copy problem. There are many local maxima and it is impossible to determine the absolute maximum. Furthermore it has been shown, see Ref. [7], that there exist a few very high maxima which have lost the vortex finding property. This was the reason why we produced for each field configuration 100 different random gauge configurations and determined the 100 corresponding relative maxima of the gauge functional (3) by the gradient ascent procedure. The projected Creutz ratios shown in Fig. 2 we got from the values of Wilson loops averaged over 100 relative maxima for each field configuraiton. Lattices of size $32^{4}$ are large enough that one can get projected Creutz ratios for distances up to $4 a$ already for a single gauge copy. Therefore we are able to plot in Fig. 3 the correlation between the relative maxima of the gauge functional and the Creutz ratio at some small distance, in our case at $r=3 a$ for the mentioned 100 gauge copies of 4 gluonic and 4 fermionic configurations. From these figures it can be seen that the Creutz ratios for the gluonic configurations fluctuate much less $(\approx 20 \%)$ than the Creutz ratios for the fermionic configurations $(\approx 50 \%)$. This seems to be a consequence of the vortex free regions which are much larger for fermionic 

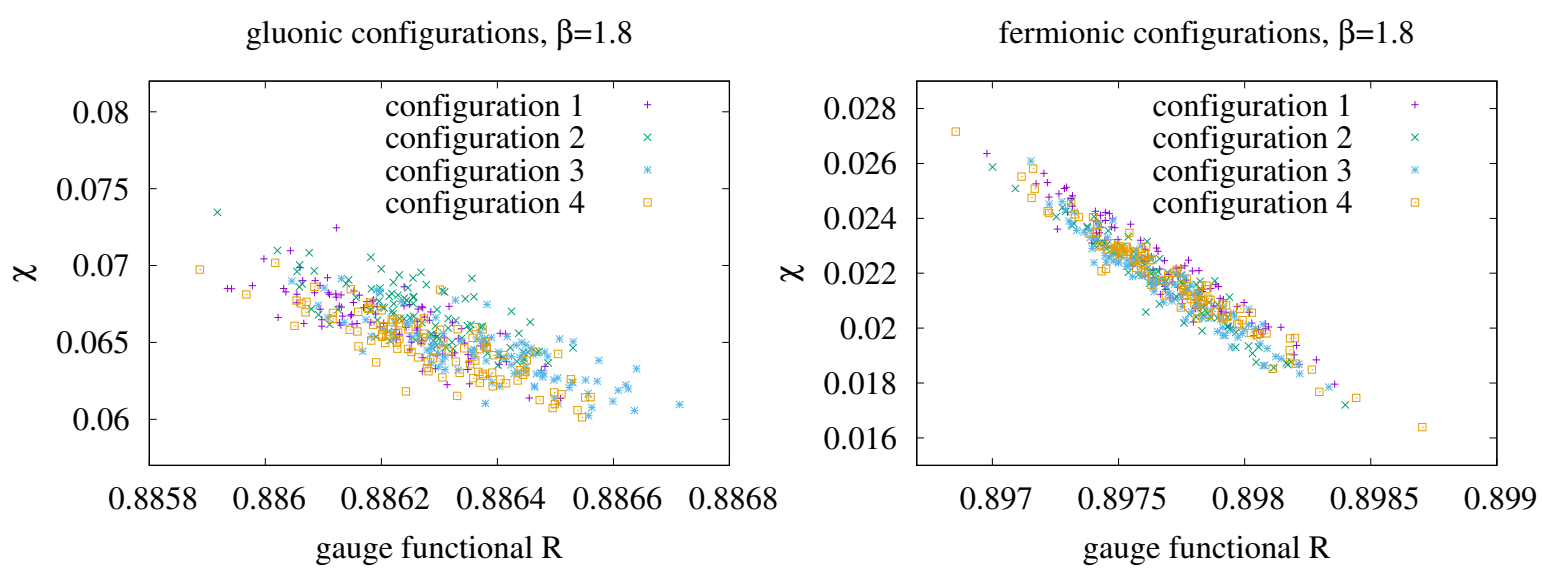

Figure 3. Correlation between the center projected Creutz ratios $\chi$ at $r=3 a$ and the maximum of the gauge functional $R$ of Eq. (3) for 100 random gauge copies of 4 gluonic and 4 fermionic configurations.

configurations, as can be seen comparing the two diagrams of Fig. 1. The average of the Creutz ratios shown in Fig. 3 leads to $\chi(3)$ of Fig. 2.

If we assume an experimental value of the string tension of $\sigma=(440 \mathrm{MeV})^{2}$ and read for the center projected gluonic configurations from Fig. 2a $\chi=0.065 \pm 5$ we get a lattice spacing $a=0.118 \pm 4 \mathrm{fm}$. For the fermionic configurations we get correspondingly $\chi=0.0223 \pm 5$ and $a=0.0669 \pm 9 \mathrm{fm}$. This value is larger then in the previous study [3]. The detailed study of this disagreement is a subject for future work.

A related interesting question concerns the breaking of the gluonic string by the fermion condensate and the possibility to get indications for a possible breaking of the string from the shape of vortices. One can compare the energy stored in the gluon string to the energy of two heavy light systems, consisting of a static color source and a dynamical fermion which screens the static charge. Such systems were recently compared in Ref. [8]. In such comparisons it is not sufficient to investigate Wilson loops. It is necessary to allow for a mixture between gluonic flux tubes and broken string states. This impossibility to detect the string breaking from Wilson loops alone may indicate that it is not possible to find an indication for the string breaking from the shape of vortices.

Acknowledgments: We thank to Vitaly Bornyakov for helpful discussions of the results. The work was supported by RFBR grants 18-32-20172 and 18-02-40126, by the STFC grant ST/P00055X/1 and N. Yu. A. acknowledges the support from the BASIS foundation.

\section{References}

1. Savvidy, G. Infrared Instability of the Vacuum State of Gauge Theories and Asymptotic Freedom. Phys.Lett. 1977, B71, 133. doi:10.1016/0370-2693(77)90759-6.

2. Greensite, J. An Introduction to the Confinement Problem; Vol. 821, Lect. Notes Phys., Springer: Berlin, Heidelberg, 2011; p. 211 pages. doi:10.1007/978-3-642-14382-3.

3. Astrakhantsev, N.Y.; Bornyakov, V.G.; Braguta, V.V.; Ilgenfritz, E.M.; Kotov, A.Y.; Nikolaev, A.A.; Rothkopf, A. Lattice study of static quark-antiquark interactions in dense quark matter. Journal of High Energy Physics 2019, 2019, 171, [arXiv:hep-lat/1808.06466]. doi:10.1007/JHEP05(2019)171.

4. Weisz, P. Continuum limit improved lattice action for pure Yang-Mills theory (I). Nuclear Physics B 1983, 212, 1 - 17. doi:https://doi.org/10.1016/0550-3213(83)90595-3.

5. Curci, G.; Menotti, P.; Paffuti, G. Symanzik's improved lagrangian for lattice gauge theory. Physics Letters B 1983, 130, 205-208. doi:10.1016/0370-2693(83)91043-2.

6. Debbio, L.D.; Faber, M.; Giedt, J.; Greensite, J.; Olejník, Š. Detection of center vortices in the lattice Yang-Mills vacuum. Phys. Rev. 1998, D58, 094501(15 pages), [hep-lat/9801027].

7. Bornyakov, V.G.; Komarov, D.A.; Polikarpov, M.I.; Veselov, A.I. P vortices, nexuses and effects of Gribov copies in the center gauges. Quantum chromodynamics and color confinement. Proceedings, 
100 International Symposium, Confinement 2000, Osaka, Japan, March 7-10, 2000, 2002, pp. 133-140, $101 \quad$ [arXiv:hep-lat/hep-lat/0210047].

102 8. Bulava, J.; Hörz, B.; Knechtli, F.; Koch, V.; Moir, G.; Morningstar, C.; Peardon, M. String breaking 103 by light and strange quarks in QCD. Phys. Lett. 2019, B793, 493-498, [arXiv:hep-lat/1902.04006]. 104 doi:10.1016/j.physletb.2019.05.018.

105 Sample Availability: Data and Fortran source codes of the algorithms are available from the authors. 